\title{
CLASIFICACION Y CARACTERIZACION DE LA FLORA Y VEGETACION DE LOS HUMEDALES DE LA COSTA DE TOLTEN (IX REGION, CHILE)
}

\author{
FLORA AND VEGETATION OF COASTAL WETLANDS NEAR TOLTEN, \\ CHILE
}

\author{
Enrique Hauenstein ${ }^{1}$, Marcos González ${ }^{1}$, Fernando Peña-Cortés ${ }^{2} \&$ Andrés Muñoz- \\ Pedreros $^{2}$
}

RESUMEN

ABSTRACT

Programas de drenado ponen en serio peligro la permanencia de los humedales de Chile. Sin embargo, la flora y vegetación de estos ecosistemas han sido poco estudiadas. Este estudio clasifica los humedales de la costa de Toltén (IX Región, Chile), determinando su flora y vegetación. De acuerdo a la clasificación de la Convención de Ramsar, se determinaron 5 tipos de humedales en el área: lagunas costeras de agua dulce, ríos y arroyos permanentes, pantanos permanentes de agua dulce, pantanos estacionales de agua dulce y humedal boscoso de agua dulce (bosque pantanoso). Se trabajó con 39 censos de vegetación de acuerdo a la metodología fitosociológica de la Escuela Zürich-Montpellier, y recolecciones al azar. La flora vascular está formada por 84 especies, en las que dominan las nativas (69\%). En el espectro biológico dominan los hemicriptófitos, seguidos en importancia por los fanerófitos y criptófitos, estos últimos representan a geófitos, helófitos e hidrófitos. Las especies más importantes en el humedal son Myrceugenia exsucca, Eleocharis macrostachya, Scirpus californicus, Juncus procerus, Anagallis alternifolia y E. pachycarpa. La tabla de vegetación ordenada muestra la presencia de 9 asociaciones vegetales: Juncetum procerii, Scirpetum californiae, Eleocharietum macrostachyaea, LotoCyperetum eragrostidae, Alismo-Sagittarietum montevidensis, Myriophylletum aquaticum, MyriophylloPotametum linguatii, Polygono-Ludwigietum peploidis y Blepharocalyo-Myrceugenietum exsuccae.

PALABRAS ClAVES: Asociación, flora, humedales, macrófitos, vegetación.

${ }^{1}$ Departamento de Ciencias Biológicas y Químicas, ${ }^{2}$ Escuela de Ciencias Ambientales, Facultad de Ciencias, Universidad Católica de Temuco. Casilla 15-D. Temuco, Chile. E-mail: ehauen@uct.cl
Drainage programs threaten many of Chile's remaining wetlands, yet the flora and vegetation of these ecosystems have yet to be thoroughly documented. This study classifies and describes the flora and vegetation of the wetlands from the coast of Tolten, IX Region, Chile. According to the Ramsar classification, five types of wetlands were determined in the area: coastal freshwater lagoons, permanent rivers and streams, freshwater marshes, seasonal freshwater and wetlands and swamp forest. Thirty-nine censuses of vegetation according to Zürich-Montpellier methodology were used. The vascular flora is formed by eightyfour species, in which the natives dominate $(69 \%)$. The hemicryptophytes dominate within the biological spectrum followed by the fanerophytes and cryptophytes. The most important species are: Myrceugenia exsucca, Eleocharis macrostachya, Scirpus californicus, Juncus procerus, Anagallis alternifolia and E. pachycarpa. The vegetation table shows the presence of nine vegetation associations: Juncetum procerii, Scirpetum californiae, Eleocharietum macrostachyaea, Loto-Cyperetum eragrostidae, AlismoSagittarietum montevidensis, Myriophylletum aquaticum, Myriophyllo-Potametum linguatii, Polygono-Ludwigietum peploidis and Blepharocalyo-Myrceugenietum exsuccae.

KEYwORDs: Asociation, flora, wetlands, macrophytes, vegetation.

\section{INTRODUCCION}

Entre los ecosistemas del planeta, los humedales destacan por su gran productividad y biodiversidad, semejándose con las selvas tropicales (Kusler et al. 1994). Al mismo tiempo, los humedales son sistemas intermedios entre ambientes permanentemente inundados y ambientes normalmente 
secos, que muestran una enorme diversidad de acuerdo a su origen, localización geográfica, su régimen acuático y químico, características del suelo o sedimento y vegetación dominante (Hauenstein et al. 1999b). Así se deja ver también en la definición que la Convención de Ramsar hace de ellos: "Extensiones de marismas, pantanos, turberas o aguas de régimen natural o artificial, permanentes o temporales, estancados o corrientes, dulces, salobres o saladas, incluyendo las extensiones de agua marina cuya profundidad en marea baja no exceda de seis metros" (MuñozPedreros \& Möller 1997).

Los humedales son decisivos para el cumplimiento de los ciclos de vida de plantas y animales, constituyen el hábitat de una gran diversidad de animales, especialmente sirven de refugio temporal a las aves migratorias. Aquellos de gran extensión tienen una función global contribuyendo a moderar los cambios climáticos, actuando como sumideros de $\mathrm{CO}_{2}$, el gas principal del efecto invernadero, como es el caso de las turberas del norte de Canadá, Alaska y Eurasia. Asimismo, las zonas húmedas amortiguan el efecto de las olas y almacenan las aguas de inundación, retienen el sedimento y reducen la contaminación; en virtud de esto último se les denominan "riñones de la naturaleza" (Kusler et al. 1994). También son importantes en la producción de alimentos, son fuente de cultivos lucrativos como el arroz silvestre, de animales de los cuales se aprovechan sus pieles, plumas y carne, de peces y mariscos, y también como fuente de forraje para los animales domésticos y silvestres.

A nivel mundial, es cada vez más clara la necesidad de conservar los humedales, debido a que constituyen ecosistemas muy diversos que representan gran valor tanto para la conservación de la diversidad biológica como para el desarrollo de las comunidades humanas asociadas a ellos (González 1993). Ha sido la Convención Ramsar, realizada en 1971 en la India, la que ha abordado la protección mundial de los humedales, junto con la formulación de planes nacionales que permitan su correcta utilización (Granizo 1997). Desde 1981 Chile es uno de los 101 países adherentes a esta convención, habiendo ya fijado algunas políticas nacionales para su conservación. De esta forma, nuestro país tiene incluidos siete humedales en la lista de aquellos de importancia internacional, de los cuales tres corresponden a la I Región y uno a las regiones II, III, V y X respectivamente, correspondiendo este último al Santuario de la Naturaleza Carlos Anwandter en el río Cruces (Muñoz-Pedreros \& Möller 1997).

La IX Región de la Araucanía es rica en humedales (IGM 1986), vale destacar sus lagos Caburgua, Colico, Villarrica y Budi importantes en el desarrollo turístico de la zona, así también lagunas y pantanos importantes para la conservación de la fauna silvestre. Muchos de estos ecosistemas han sido poco estudiados y por lo mismo no se les ha dimensionado en sus reales potencialidades, por ejemplo, en el ecoturismo. Muchos de ellos son considerados, en especial por los agricultores, como ambientes perdidos que hay que drenar y recuperar para el cultivo. De esta forma, en varios sectores de la región se están aplicando programas, incentivados y financiados con fondos estatales, de "recuperación" de esos terrenos para la agricultura, por lo que gran parte de estos ecosistemas se perderán irremediablemente. Según Conaf \& Conama (1999) sólo en la provincia de Cautín existen 17.625,2 ha con humedales (palustre, ribereño y lacustre), de los cuales el $35,8 \%$ se encuentra en el Borde Costero que comprende las comunas de Carahue, Puerto Saavedra, Teodoro Schmidt y Toltén. Este territorio es además una zona socioeconómicamente muy deprimida y con una población mayoritariamente mapuche. Destacan en el conjunto del área los hualves o bosques pantanosos de temo y pitra del río Mahuidanchi, ubicados en el extremo noreste de Toltén, mencionados en el libro rojo de los sitios prioritarios para la conservación de la diversidad biológica de Chile (Muñoz et al. 1996); a pesar de ello, están siendo intervenidos con el objeto de drenarlos y obtener suelos aptos para la agricultura (Hauenstein et al. 1999b, 2001).

El presente estudio pretendió establecer los tipos de humedales presentes en las cuencas hidrográficas de los ríos Boldo y Boroa en la comuna de Toltén, asimismo, determinar su diversidad florística y vegetacional.

\section{AREA DE ESTUDIO}

La comuna de Toltén se ubica aproximadamente a $90 \mathrm{~km}$ al sur-poniente de la ciudad de Temuco, 
provincia de Cautín, IX Región de la Araucanía ( $\left.38^{\circ} 52^{\prime} \mathrm{S}-73^{\circ} 18^{\prime} \mathrm{W}\right)$, siendo la altitud del área de humedales de entre 0 y $25 \mathrm{msnm}$. El área específica estudiada comprende la cuenca hidrográfica del río Boroa y la parte inferior de la cuenca del río Boldo. Según Di Castri \& Hajek (1976) el clima del sector es oceánico con influencia mediterránea. Según Koeppen (1931) corresponde al tipo Cfb (templado húmedo de verano fresco). Su temperatura media anual es de $12^{\circ} \mathrm{C}$ y su precipitación media anual de 1.553 $\mathrm{mm}$. Los terrenos son preferentemente de uso forestal y el resto corresponde a terrazas fluviales con predominio de praderas y vegas de uso agro-ganadero (Hauenstein et al. 2001).

Es una comuna socioeconómicamente muy deprimida; abarca una superficie de $936,8 \mathrm{~km}^{2}$ y posee una población de más de 12.000 habitantes, de los cuales el $87 \%$ son campesinos con un alto componente mapuche. La economía de la zona se basa en actividades agrícolas, ganaderas, pesqueras y forestales. En cuanto a recursos naturales es especialmente rica en humedales, tanto en abundancia como en variedad, pero no se les ha inventariado y se desconoce las características de sus aguas y sustrato, de su flora y fauna asociada y de sus potencialidades como recurso para las comunidades humanas aledañas. Por otra parte, los hualves del río Mahuidanchi, ubicados en el extremo noreste del sector, están mencionados en el libro rojo de los sitios prioritarios para la conservación de la diversidad biológica de Chile (Muñoz et al. 1996).

\section{MATERIALES Y METODOS}

En los meses de julio 1999 a enero 2000 se realizaron 4 excursiones, en las cuales se trabajó en base a inventarios fitosociológicos y recolecciones al azar. Se utilizaron parcelas de $4 \mathrm{~m}^{2}$ para la vegetación herbácea, de $25 \mathrm{~m}^{2}$ para matorral y de $100 \mathrm{~m}^{2}$ para la arbórea (bosques pantanosos). Las especies no identificadas en terreno fueron determinadas en el laboratorio, utilizando para tal efecto literatura especializada. Para cada especie se consideró su nombre científico, familia botánica, nombre común, forma de vida y origen fitogeográfico. Esta información fue compilada en un catálogo florístico (Tabla I). Las especies recolectadas quedaron depositadas en el herbario del Departamento de Ciencias Biológicas y Químicas de la Facultad de Ciencias, Universidad Católica de Temuco.

La clasificación, nomenclatura y origen geográfico de las especies se basó principalmente en Marticorena \& Quezada (1985) y Matthei (1995). Las formas de vida en Ellenberg \& Mueller-Dombois (1966), detallando las formas de vida de los criptófitos según Ramírez \& Stegmeier (1982). La determinación del grado de intervención antrópica del área se obtuvo sobre la base de la propuesta de Hauenstein et al. (1988), que considera el origen fitogeográfico y las formas de vida como medida de esta forma de perturbación, es decir, la relación de especies nativas versus introducidas y la abundancia de hemicriptófitos. Se levantaron 39 relevamientos fitosociológicos y las tablas fitosociológicas se elaboraron de acuerdo a la metodología europea de tabulación (BraunBlanquet 1964), explicitada en Ramírez \& Westermeier (1976). En ellas, y en cada censo, se anotó para cada especie su cobertura en porcentaje; para valores menores a $1 \%$ se utilizó el signo "+" cuando había varios individuos y el signo " $r$ " cuando existía uno solo. Esta Tabla inicial se ordenó primero horizontalmente mediante la frecuencia de las especies en orden decreciente y luego verticalmente utilizando especies diferenciales que se excluyen mutuamente. De las tablas anteriores se obtuvo una tabla ordenada (Tabla II) que incluye el valor de importancia de cada especie, el que se obtuvo sumando la frecuencia (porcentaje de los censos en que la especie está presente) y la cobertura relativas de acuerdo a Wikum \& Shanholtzer (1978). En esta Tabla se aprecian las agrupaciones de especies que corresponden a las diferentes asociaciones.

La tipificación de los humedales se realizó de acuerdo a la clasificación de la Convención de Ramsar, presentada en Muñoz-Pedreros \& Möller (1997).

\section{RESULTADOS}

\section{CLASIFICACIÓN DE LOS HUMEDALES}

Los humedales presentes en el área de estudio corresponden a humedales naturales, costeros, continentales y ribereños, de los siguientes tipos:

(K) Lagunas costeras de agua dulce. Es el caso de las lagunas Patagua y Tromén. 
(M) Ríos y arroyos permanentes. Es el caso de los ríos Boldo, Boroa y Queule.

(Tp) Pantanos, esteros o charcas permanentes de agua dulce, con vegetación emergente en agua por lo menos durante la mayor parte del período de crecimiento. Abundantes en el área de Toltén sur.

(Ts) Pantanos, esteros o charcas estacionales de agua dulce, praderas inundadas estacionalmente y pantanos de ciperáceas. Abundantes en el área de Toltén sur.

(Xf) Humedales boscosos de agua dulce. Corresponden específicamente a los bosques pantanosos de temo y pitra, abundantes en el sector de Mahuidanchi, al noreste de Toltén, y a orillas de los ríos Boldo y Boroa.

FLORA

Los resultados del estudio florístico realizado en estos humedales muestran la presencia de 84 especies de plantas vasculares (Tabla I). El grupo taxonómico mejor representado es el de las dicotiledóneas (Magnoliopsida) con un 66\%, le siguen las monocotiledóneas (Liliopsida) con un $30 \%$, existiendo sólo un $4 \%$ de pteridófitos y ausencia de gimnospermas (Fig. 1).

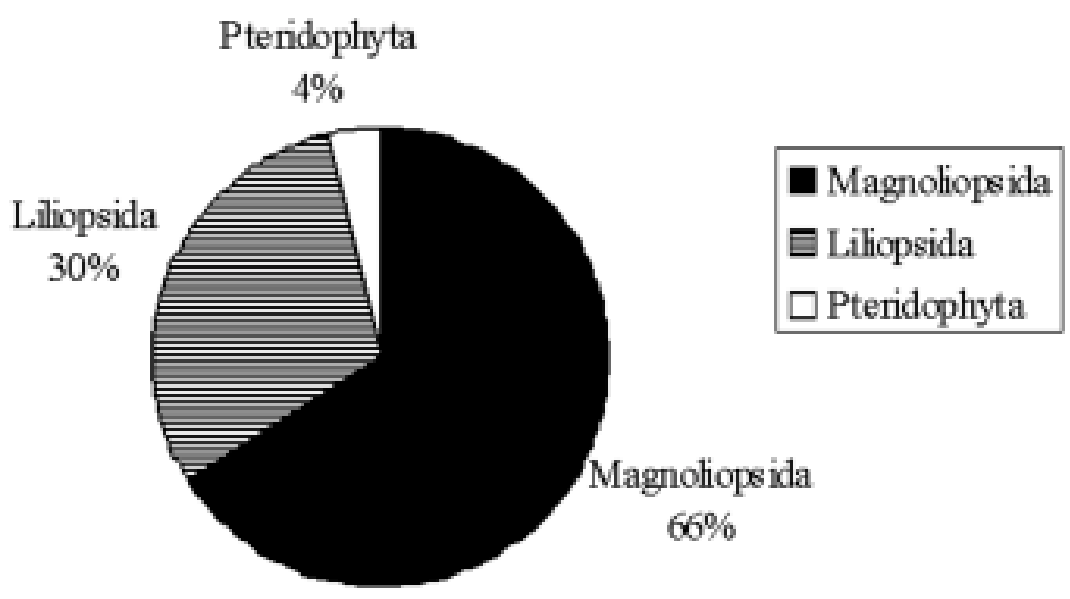

Figura 1. Distribución taxonómica (en porcentaje) de la flora vascular de los humedales de Toltén.

La forma de vida predominante corresponde a los hemicriptófitos (37 spp), que comprende hierbas y malezas perennes adaptadas a soportar el ramoneo y pisoteo de los animales, con un número similar de especies nativas e introducidas (Fig. 2). En segundo lugar destacan las especies leñosas (30 spp), representadas por fanerófitos (árboles), nanofanerófitos (arbustos) y fanerófitos trepadores, epífitos y parásitos vasculares, con un mayor número de especies nativas que introducidas. Luego se encuentran los criptófitos con 11 especies, que incluye a helófitos, hidrófitos y geófitos, donde también predominan las nativas, y finalmente, están los terófitos y caméfitos con 4 y 2 especies respectivamente.

Globalmente, el porcentaje de especies nativas (69\%) supera a las alóctonas (29\%) y cosmopolitas (2\%) (Fig. 3). 
Humedales centro-sur de Chile: Hauenstein, E. ET AL.

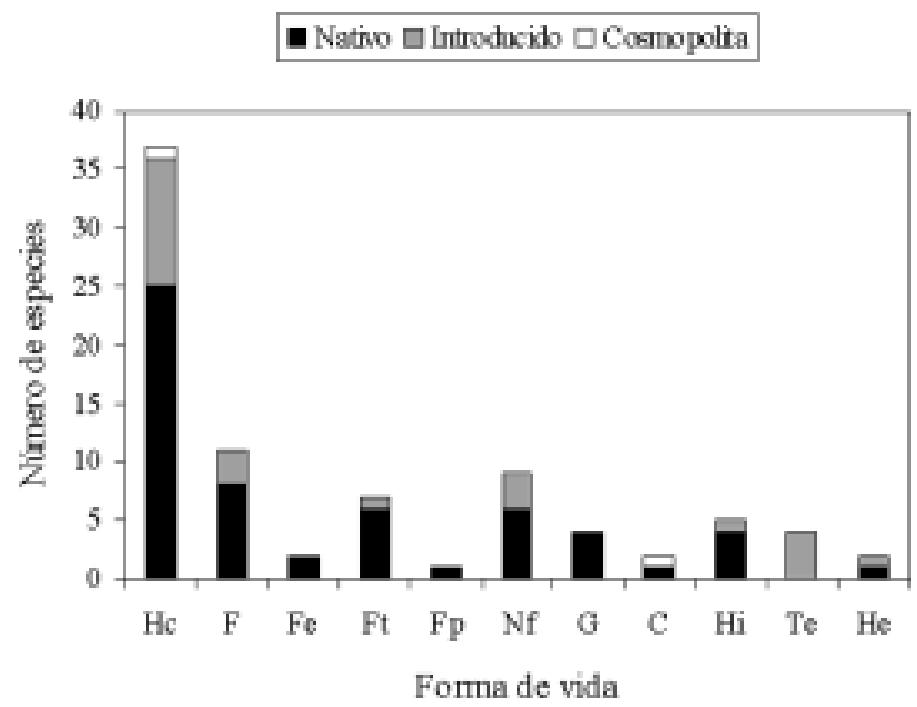

FigURA 2. Espectro biológico y origen geográfico de la flora vascular de los humedales de Toltén (Hc = Hemicriptófito, $\mathrm{F}=$ Fanerófito, $\mathrm{Fe}=$ Fanerófito epífito, $\mathrm{Ft}=$ Fanerófito trepador, $\mathrm{Fp}=$ Fanerófito parásito, $\mathrm{Nf}=$ Nanofanerófito, $\mathrm{G}=$ Geófito, $\mathrm{C}=$ Caméfito, $\mathrm{Te}=$ Terófito, $\mathrm{He}=$ Helófito $)$.

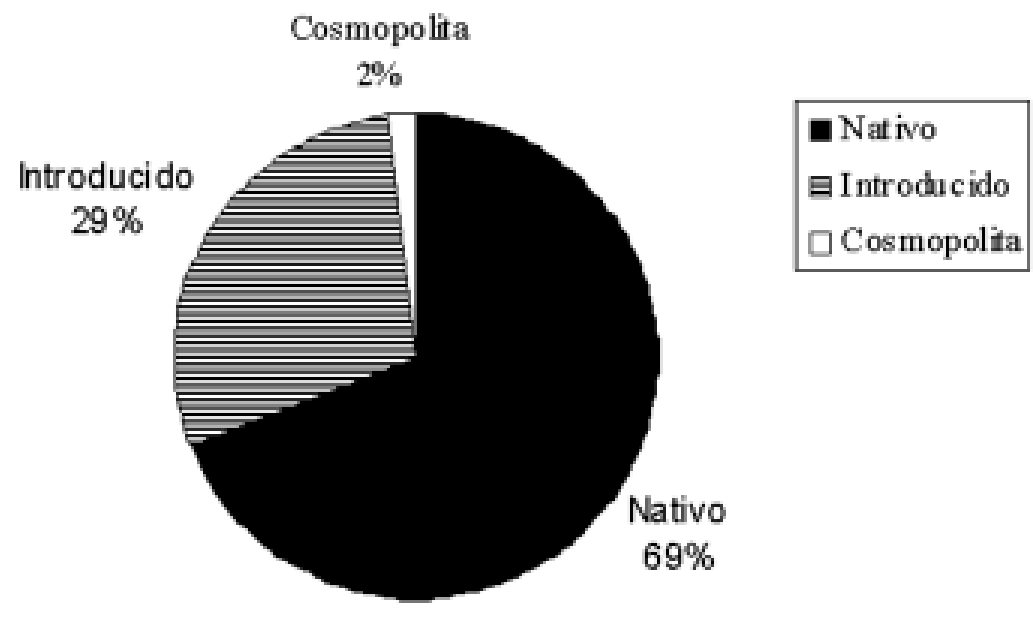

FIgURA 3. Origen geográfico de la flora vascular de los humedales de Toltén. 
TABLa I. Catálogo de la flora vascular de los humedales de Toltén.

Clase/Nombre Cient fico

FILICOPSIDA

Blechnum blechnoides Keyserl.

Blechnum cordatum (Desv.) Hieron.

Hymenophyllum dentatum Cav.

\section{MAGNOLIOPSIDA}

Amomyrtus luma (Mol.) Legr. et Kaus. Anagallis alternifolia Cav.

Aster vahlii (Gaud.) H. et A.

Azara serrata $\mathrm{R}$. et P.

Azara microphylla Hook. f.

Baccharis sagittalis (Less.) DC.

Berberis darwinii Hook.

Blepharocalyx cruckshanksii (H. et A.) Nied.

Boquila trifoliolata (DC.) Dcne.

Calystegia sepium (L.) R. Br.

Callitriche palustris $\mathrm{L}$.

Cardamine nasturtioides Bertero

Cirsium vulgare (Savi) Ten.

Cissus striata R. et P.

Dichondra sericea $\mathrm{Sw}$.

Drimys winteri J.R. et G. Forster

Galium aparine $\mathrm{L}$.

Geranium core-core Steud.

Gratiola peruviana $\mathrm{L}$.

Hydrocotyle modesta Cham. et Shlecht.

Hypochaeris radicata $\mathrm{L}$.

Laurelia sempervirens (R. et P.) Tul.

Leontodon saxatilis Lam.

Lepidoceras chilensis (Mol.) Kuijt

Lotus uliginosus Schkuhr

Ludwigia peploides (Kunth) P.H. Raven

Luma gayana (Barn.) Burret

Lupinus arboreus Sims

Myriophyllum aquaticum (Vell.) Verdc.

Muehlenbeckia hastulata (J.E. Sm.) Johnst.

Myoschilos oblonga R. et P.

Myrceugenia chrysocarpa (Berg) Kausel

Myrceugenia exsucca (DC.) Berg

Nertera granadensis (Mutis ex L.f.) Druce

Nothofagus dombeyi (Mirb.) Oerst.

Pilea elliptica Hook.f.

Plantago major L.

Polygonum hydropiperoides Michx.

Potentilla anserina L.

Prunella vulgaris L.

Pseudopanax valdiviensis (Gay) Seem. ex Reiche

Ranunculus repens L.

Rorippa nasturtium-aquaticum (L.) Hayek

Rosa rubiginosa L.

Rubus constrictus Muell. et Lef.

Rumex acetosella $\mathrm{L}$.

Salix babylonica $\mathrm{L}$.
Familia

Nombre Comøn

Fv Of

$\begin{array}{llcl}\text { Blechnaceae } & \text { Iquide } & \text { Hc } & \text { N } \\ \text { Blechnaceae } & \text { Costilla de vaca } & \text { Hc } & \text { N } \\ \text { Hymenophyllaceae } & \text { Helecho pel cula } & \text { Fe } & \text { N }\end{array}$

\begin{tabular}{|c|c|c|}
\hline Myrtaceae & Luma & $\mathrm{F}$ \\
\hline Primulaceae & Pimpinela & $\mathrm{Hc}$ \\
\hline Asteraceae & Margarita del pantano & $\mathrm{G}$ \\
\hline Flacourtiaceae & Corcolø & $\mathrm{Nf}$ \\
\hline Flacourtiaceae & Chin chin & $\mathrm{Nf}$ \\
\hline Asteraceae & $\begin{array}{l}\text { Verbena de tres } \\
\text { esquinas }\end{array}$ & $\mathrm{C}$ \\
\hline Berberidaceae & Michay & $\mathrm{Nf}$ \\
\hline Myrtaceae & Temu & $\mathrm{F}$ \\
\hline Lardizabalaceae & Voqui blanco & $\mathrm{Ft}$ \\
\hline Convolvulaceae & Carricillo & $\mathrm{Ft}$ \\
\hline Callitrichaceae & Huenchec & $\mathrm{Hi}$ \\
\hline Brassicaceae & Berro & $\mathrm{Hi}$ \\
\hline Asteraceae & Cardo negro & $\mathrm{Te}$ \\
\hline Vitaceae & Voqui negro & $\mathrm{Ft}$ \\
\hline Convolvulaceae & Oreja de rat $n$ & $\mathrm{Hc}$ \\
\hline Winteraceae & Canelo & $\mathrm{F}$ \\
\hline Rubiaceae & s.n. & $\mathrm{Hc}$ \\
\hline Geraniaceae & Core-core & $\mathrm{Hc}$ \\
\hline Scrophulariaceae & Contrahierba,Huilhue & $\mathrm{He}$ \\
\hline Apiaceae & Sombrerito de agua & $\mathrm{Hc}$ \\
\hline Asteraceae & Hierba del chancho & $\mathrm{Hc}$ \\
\hline Monimiaceae & Laurel & $\mathrm{F}$ \\
\hline Asteraceae & Chinilla & $\mathrm{Hc}$ \\
\hline Loranthaceae & Quintral del temu & Fp \\
\hline Fabaceae & Alfalfa chilota & $\mathrm{Hc}$ \\
\hline Onagraceae & Melilucul & $\mathrm{Hi}$ \\
\hline Myrtaceae & Chin chin & $\mathrm{F}$ \\
\hline Fabaceae & Chocho & $\mathrm{Nf}$ \\
\hline Haloragaceae & Pinito de agua & $\mathrm{Hi}$ \\
\hline Polygonaceae & Quilo & $\mathrm{Ft}$ \\
\hline Santalaceae & Orocoipo & $\mathrm{Nf}$ \\
\hline Myrtaceae & Luma blanca & $\mathrm{Nf}$ \\
\hline Myrtaceae & Pitra & $\mathrm{F}$ \\
\hline Rubiaceae & Chaquirita del monte & $\mathrm{Hc}$ \\
\hline Fagaceae & Coihue & $\mathrm{F}$ \\
\hline Urticaceae & s.n. & $\mathrm{Hc}$ \\
\hline Plantaginaceae & LlantØ & $\mathrm{Hc}$ \\
\hline Polygonaceae & Duraznillo de agua & $\mathrm{Hc}$ \\
\hline Rosaceae & Canelilla & $\mathrm{Hc}$ \\
\hline Lamiaceae & Hierba mora & $\mathrm{C}$ \\
\hline Araliaceae & Curaco & $\mathrm{Ft}$ \\
\hline Ranunculaceae & Bot $\mathrm{n}$ de oro & $\mathrm{Hc}$ \\
\hline Brassicaceae & Berro & $\mathrm{He}$ \\
\hline Rosaceae & Mosqueta & $\mathrm{Nf}$ \\
\hline Rosaceae & Murra & $\mathrm{Nf}$ \\
\hline Polygonaceae & Vinagrillo & $\mathrm{Hc}$ \\
\hline Salicaceae & Sauce llor $\mathrm{n}$ & $\mathrm{F}$ \\
\hline
\end{tabular}


Humedales centro-sur de Chile: Hauenstein, E. ET AL.

TABLA I: continuación

Clase/Nombre Cient fico Familia

Nombre Coman

Fv Of

Salix caprea L.

Salix viminalis $\mathrm{L}$.

Sarmienta scandens (J.D.Brandis) Pers.

Scutellaria racemosa Pers.

Senecio fistulosus Poepp. ex Less.

Tepualia stipularis (H. et A.) Griseb.

Trifolium repens $\mathrm{L}$.

Veronica serpyllifolia $\mathrm{L}$.

Verbena bonariensis $L$.

\section{LILIOPSIDA}

Agrostis capillaris L.

Alisma plantago-aquatica $\mathrm{L}$.

Carex acutata Boott

Carex brongniartii Kunth

Carex fuscula D. Urv. var. fuscula

Cyperus eragrostis Lam.

Cyperus eragrostis Lam. var. compactus

Chusquea quila Kunth

Distichlis spicata (L.) Greene

Eleocharis macrostachya Britton

Eleocharis pachycarpa Desv.

Fascicularia bicolor ( R. et P.) Mez

Holcus lanatus L.

Juncus bufonius L.

Juncus cyperoides Lah.

Juncus dombeyanus J. Gay ex Lah.

Juncus microcephalus H.B.K.

Juncus procerus E.Meyer

Luzuriaga radicans $\mathrm{R}$.et $\mathrm{P}$.

Phragmites australis (Cav.)Trin. ex Steud.

Poa annua $\mathrm{L}$.

Potamogeton linguatus Hagstr.

Sagittaria montevidensis Cham. et Schlecht.

Scirpus californicus (C.A.Mey.) Steud.

Scirpus inundatus (R.Br.) Poir.

Salicaceae
Salicaceae
Gesneriaceae
Lamiaceae
Asteraceae
Myrtaceae
Fabaceae
Scrophulariaceae
Verbenaceae

Sauce cabruno

Sauce mimbre

Medallita

s.n.

Hualtata

Tерœ

TrØol blanco

Ver nica europea

Verbena

\begin{tabular}{llcc} 
Poaceae & Chøica & $\mathrm{Hc}$ & $\mathrm{N}$ \\
Alismataceae & Llantø de agua & $\mathrm{Hc}$ & $\mathrm{I}$ \\
Cyperaceae & Cortadera & $\mathrm{Hc}$ & $\mathrm{N}$ \\
Cyperaceae & Cortadera & $\mathrm{G}$ & $\mathrm{N}$ \\
Cyperaceae & Cortadera & $\mathrm{Hc}$ & $\mathrm{N}$ \\
Cyperaceae & Cortadera & $\mathrm{Hc}$ & $\mathrm{N}$ \\
Cyperaceae & Cortadera & $\mathrm{Hc}$ & $\mathrm{N}$ \\
Poaceae & Quila & $\mathrm{NF}$ & $\mathrm{N}$ \\
Poaceae & Grama brava & $\mathrm{Hc}$ & $\mathrm{N}$ \\
Cyperaceae & Rume & $\mathrm{Hc}$ & $\mathrm{N}$ \\
Cyperaceae & Rume & $\mathrm{Hc}$ & $\mathrm{N}$ \\
Bromeliaceae & Chupalla & $\mathrm{Fe}$ & $\mathrm{N}$ \\
Poaceae & Pasto dulce & $\mathrm{Te}$ & $\mathrm{I}$ \\
Juncaceae & Junquillo & $\mathrm{Te}$ & $\mathrm{I}$ \\
Juncaceae & Ihua-Ihua & $\mathrm{Hc}$ & $\mathrm{N}$ \\
Juncaceae & Junquillo & $\mathrm{Hc}$ & $\mathrm{N}$ \\
Juncaceae & Junquillo & $\mathrm{Hc}$ & $\mathrm{N}$ \\
Juncaceae & Junquillo & $\mathrm{Hc}$ & $\mathrm{N}$ \\
Philesiaceae & Azahar del monte & $\mathrm{Ft}$ & $\mathrm{N}$ \\
Poaceae & Carrizo & $\mathrm{Hc}$ & $\mathrm{Co}$ \\
Poaceae & Piojillo & $\mathrm{Te}$ & $\mathrm{I}$ \\
Potamogetonaceae & Huiro & $\mathrm{Hi}$ & $\mathrm{N}$ \\
Alismataceae & Lengua de vaca & $\mathrm{Hc}$ & $\mathrm{N}$ \\
Cyperaceae & Totora & $\mathrm{G}$ & $\mathrm{N}$ \\
Cyperaceae & Can can & $\mathrm{G}$ & $\mathrm{N}$ \\
\hline & & & \\
\hline & & &
\end{tabular}

$\mathrm{FV}=$ Forma de vida, $\mathrm{OF}=$ Origen fitogeogr Afico, $\mathrm{F}=$ Faner fito, $\mathrm{NF}=$ Nanofaner fito, $\mathrm{Fe}=$ Faner fito ep fito, $\mathrm{Fp}=$ Faner fito par/sito, $\mathrm{Ft}=$ Faner fito trepador, $\mathrm{C}=$ CamØito, $\mathrm{Hc}=$ Hemcript fito, $\mathrm{G}=\mathrm{Ge}$ fito, $\mathrm{Hi}=$ Hidr fito, $\mathrm{Te}=$ Ter fito, $\mathrm{N}=$ Nativo, $\mathrm{I}=$ Introducido, $\mathrm{Co}=$ Cosmopolita . 


\section{VEGETACIÓN}

Los resultados del estudio fitosociológico indican la presencia en estos humedales de las siguientes asociaciones vegetales, cuya estructura florística se muestra en la Tabla II:

1. Pradera húmeda de junquillo (Juncetum procerii Oberdorfer 1960).

Representada por los inventarios 1-10 y 37 . Es una comunidad pratense antropogénica, rica en especies, donde predominan las alóctonas. La especie dominante es Juncus procerus, especie nativa, junto a Lotus uliginosus, Holcus lanatus, Dichondra sericea y Agrostis capillaris, todas alóctonas.

2. Pradera de rüme o quilmén (Eleocharietum macrostachyae Contreras, Verdugo, San Martín \& Ramírez 1991).

Inventarios 14-17 y 30-31. Corresponde a una pradera muy húmeda que ocupa sólo algunos sectores planos inundados, pero con un sustrato profundo; presenta pocas especies y las dominantes, Eleocharis macrostachya y E. pachycarpa, son de gran palatabilidad para el ganado.

3. Comunidad de cortadera (Loto-Cyperetum eragrostidae San Martín, Medina, Ojeda \& Ramírez 1993).

Inventarios 11-13. Corresponde a una asociación palustre donde dominan las especies nativas Cyperus eragrostis y Carex acutata, las que en su máximo crecimiento pueden alcanzar hasta $1 \mathrm{~m}$ de altura. Esta comunidad se desarrolla en franjas bien delimitadas próximas al totoral y a la pradera de junquillo.

\section{Totoral (Scirpetum californiae Añazco 1978).}

Inventarios 18-20. Esta asociación palustre es abundante en el lugar y se caracteriza por colonizar diferentes biotopos acuáticos, tanto lénticos como lóticos. Presenta pocas especies, con una alta cobertura de Scirpus californicus (totora), hierba helófita nativa que puede superar los $2 \mathrm{~m}$ de altura y que posee un robusto rizoma del cual nacen culmos aéreos que sólo viven una temporada.
5. Comunidad de lengua de vaca (AlismoSagittarietum montevidensis San Martín, Medina, Ojeda \& Ramírez 1993).

Inventarios 25-26. Asociación que se ubica habitualmente en una franja vegetacional más próxima al agua que el Scirpetum californiae, el que generalmente la desplaza al aumentar el nivel del sedimento. La especie dominante es Sagittaria montevidensis y la secundaria es Alisma plantagoaquatica, la que no aparece representada en los dos inventarios antes señaladas, debido seguramente a una situación de deterioro de la comunidad. Según San Martín et al. (1993) en esta asociación es importante también Egeria densa, especie que no fue prospectada en el área de estudio.

6. Comunidad de pasto pinito (Myriophylletum aquaticum Medina 1988).

Inventarios 22, 24, 32. Esta comunidad sumergida coloniza aguas someras lénticas, con sustrato fangoso. Es una comunidad casi pura, cuya especie principal es Myriophyllum aquaticum que muestra alta cobertura.

7. Comunidad de huiro (Myriophyllo-Potametum linguatii Barrera \& Ramírez 1986).

Inventarios 21, 23, 28, 29. Asociación que prospera en ambientes lénticos empozados, con sustrato fangoso y con profundidades que pueden llegar a los $2 \mathrm{~m}$. La especie más importante es Potamogeton linguatus, que posee dos tipos de hojas: natantes y sumergidas. La otra especie característica de esta comunidad es Myriophyllum aquaticum.

8. Comunidad de pasto de la rana (PolygonoLudwigietum peploidis Steubing, Ramírez \& Alberdi 1980).

Inventario 27. Comunidad de hoja natante que parte desde las orillas de sectores fangosos (pequeñas lagunas) hasta el centro del cuerpo de agua. La especie más importante es Ludwigia peploides, con alta cobertura, que también presenta heterofilia, con hojas acuáticas natantes redondeadas y las que se desarrollan en ambientes más secos o pantanosos son de forma elongada. 
TABLA II. Tabla ordenada de la vegetación de los humedales de Toltén, con valores de importancia (V.I.).

\begin{tabular}{|c|c|c|c|c|c|c|c|c|c|c|c|c|c|c|c|c|c|c|c|c|c|c|c|c|c|c|c|c|c|c|c|c|c|c|}
\hline \multirow[t]{2}{*}{ ESPECIES } & \multicolumn{10}{|c|}{ A } & \multicolumn{3}{|c|}{ B } & \multicolumn{5}{|c|}{$\begin{array}{c}\text { C } \\
\text { INVENTARIOS }\end{array}$} & \multicolumn{3}{|c|}{$\mathrm{D}$} & \multicolumn{2}{|c|}{$\mathrm{E}$} & $\mathrm{F}$ & \multicolumn{2}{|l|}{ G } & \multicolumn{3}{|l|}{$\mathrm{H}$} & \multicolumn{4}{|c|}{ I } & \multirow{2}{*}{ V.I. } \\
\hline & 1 & 2 & 3 & 4 & 5 & 6 & 78 & 8 & 91 & 10 & 3711 & 12 & 13 & 14 & 151 & 16 & $17 \quad 30$ & 31 & 18 & 192 & 202 & 2224 & 24322 & 2526 & 627 & 21 & 232 & $28 \quad 29$ & 33 & 3435 & 536 & 38 & 39 & \\
\hline Especies diferenciales & & & & & & & & & & & & & & & & & & & & & & & & & & & & & & & & & & \\
\hline Juncus procerus & 15 & 40 & 25 & 10 & 20 & 10 & 101 & 10 & $5 \quad 5$ & 501 & 1010 & 10 & 10 & + & & & & & & & & & & & & & & & & & & & & 10.2 \\
\hline Lotus uliginosus & 5 & 10 & 5 & 15 & 10 & 5 & 10 & 5 & 55 & 5 & +5 & + & 5 & + & 5 & + & 5 & & & & + & ++ & + & & & & + & & & & + & + & & 8.9 \\
\hline Juncus dombeyanus & & & & & & & & & & + & + & 10 & 30 & 10 & & + & & & & & & 105 & 5 & & & 10 & 5 & + & & & & & & 5.0 \\
\hline Cyperus eragrostis & & & & & & & & & & & 60 & 40 & 10 & & & & & 5 & 20 & 5 & & & + & ++ & ++ & + & & + & & & & & & 7.0 \\
\hline Carex acutata & & & & & & & & & & & 10 & 40 & 30 & & 5 & & & & 10 & 5 & 5 & & + & & & & + & & & & + & + & & 5.7 \\
\hline Eleocharis macrostachya & & & & & & & & & & + & & & & 20 & 101 & 10 & 6095 & 90 & & & & & + & & & & + & & & & & & & 10.5 \\
\hline Eleocharis pachycarpa & 5 & + & + & + & & & 101 & 101 & 101 & 10 & + & & & 30 & 305 & 50 & $10+$ & 5 & & & & & & & & & & & & & & & & 8.5 \\
\hline Polygonum hydropiperoides & & & & & & & & & & & & & & 10 & 151 & 15 & & & 10 & 10 & & ++ & ++ & & & + & + & & & & & & & 4.3 \\
\hline Scirpus californicus & & & & & & & & & & & & & & & & & & + & 60 & 808 & 801 & 1010 & $10+$ & & 10 & 20 & 10 & & & & & & & 10.2 \\
\hline Myriophyllum aquaticum & & & & & & & & & & & & & & & & & & & & & & 5565 & 6555 & ++ & + & & + & & & & & & & 6.3 \\
\hline Sagittaria montevidensis & & & & & & & & & & & & & & + & 5 & & 10 & & + & & & $+\quad+$ & $+\quad 6$ & 6060 & 0 & 5 & 5 & & & & & & & 6.6 \\
\hline Ludwigia peploides & & & & & & & & & & & & & & & & & & & & & & & 5 & + & +80 & & & & & & & & & 3.1 \\
\hline Potamogeton linguatus & & & & & & & & & & & & & & & & & & & & & & + & & & & 25 & 407 & 7090 & & & & & & 7.4 \\
\hline Myrceugenia exsucca & & & & & & & & & & & & & & & & & & & & & & & & & & & & & 504 & 4060 & 060 & 60 & 60 & 10.7 \\
\hline Blepharocalix cruckshanksii & & & & & & & & & & & 10 & & & & & & & & & & & & & & & & & & 50 & 605 & 30 & + & 30 & 6.8 \\
\hline Especies acompaæantes & & & & & & & & & & & & & & & & & & & & & & & & & & & & & & & & & & \\
\hline Anagallis alternifolia & 5 & 5 & 5 & + & & & 406 & 607 & 701 & 10 & + & & & 5 & 10 & 5 & + & + & & & + & & & & & & & & & & & & & 9.8 \\
\hline Hydrocotyle modesta & 10 & 10 & 5 & 5 & & 10 & 10 & + & ++ & + & ++ & + & + & 5 & 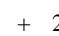 & 20 & & + & & & + & & & & & & & & & & + & + & & 7.4 \\
\hline Dichondra sericea & 30 & 20 & 20 & 20 & & & 10 & 5 & $5 \quad 1$ & 101 & 10 & & + & & & & + & & & & & & & & & & & & & & & & & 6.3 \\
\hline Agrostis capillaris & 10 & 5 & 20 & 10 & 30 & 25 & & & & & 60 & & & & & & + & + & & & & & & & & & & & & & & & & 4.8 \\
\hline Holcus lanatus & 5 & 10 & 10 & 10 & 10 & 5 & + & 5 & + & & + & & & & & & & & & & & & & & & & & & & & & & & 4.1 \\
\hline Rubus constrictus & & & & 5 & + & & & & & & +5 & + & + & & & & & & & & & & & & & & & & + & ++ & + & + & + & 3.6 \\
\hline Gratiola peruviana & & & & & & & & & & + & 5 & + & & 5 & + & + & + & & & & & & & & & & & & & & + & + & & 2.7 \\
\hline Ranunculus repens & 5 & & 10 & 20 & 10 & & & & & & + & & & & & & & & & & & & & & & & & & & & & & & 2.5 \\
\hline Distichlis spicata & & & & & & & 10 & 5 & +5 & 5 & 5 & & + & & & & + & & & & & & & & & & & & & & & & & 2.5 \\
\hline Carex fuscula & + & + & + & + & & & & + & $5+$ & + & & & & & & & + & & & & & & & & & & & & & & & & & 2.3 \\
\hline Blechnum cordatum & & & & & & & & & & & $+r$ & + & + & & & & & & & & & & & & & & & & & + & + & + & + & 2.2 \\
\hline Luma gayana & & & & & & & & & & & & & & & & & & & & & & & & & & & & & + & +5 & 5 & 5 & 10 & 2.2 \\
\hline Leontodon saxatilis & + & + & + & + & 5 & + & & & + & & & & & & & & & & & & & & & & & & & & & & & & & 2.1 \\
\hline Scirpus inundatus & & & & & & & & & & & & & & & & & + & + & + & & & & + & & & & & & & & + & + & & 1.9 \\
\hline Prunella vulgaris & + & & + & + & + & + & & & & & + & & & & & & & & & & & & & & & & & & & & & & & 1.7 \\
\hline Luzuriaga radicans & & & & & & & & & & & & & & & & & & & & & & & & & & & & & + & ++ & + & + & + & 1.7 \\
\hline Rumex acetosella & & + & + & & + & 20 & & & & & & & & & & & & & & & & & & & & & & & & & & & & 1.6 \\
\hline Drimys winteri & & & & & & & & & & & & & & & & & & & & & & & & & & & & & & ++ & 5 & 5 & + & 1.6 \\
\hline Trifolium repens & + & + & & & + & 15 & & & & & & & & & & & & & & & & & & & & & & & & & & & & 1.5 \\
\hline
\end{tabular}




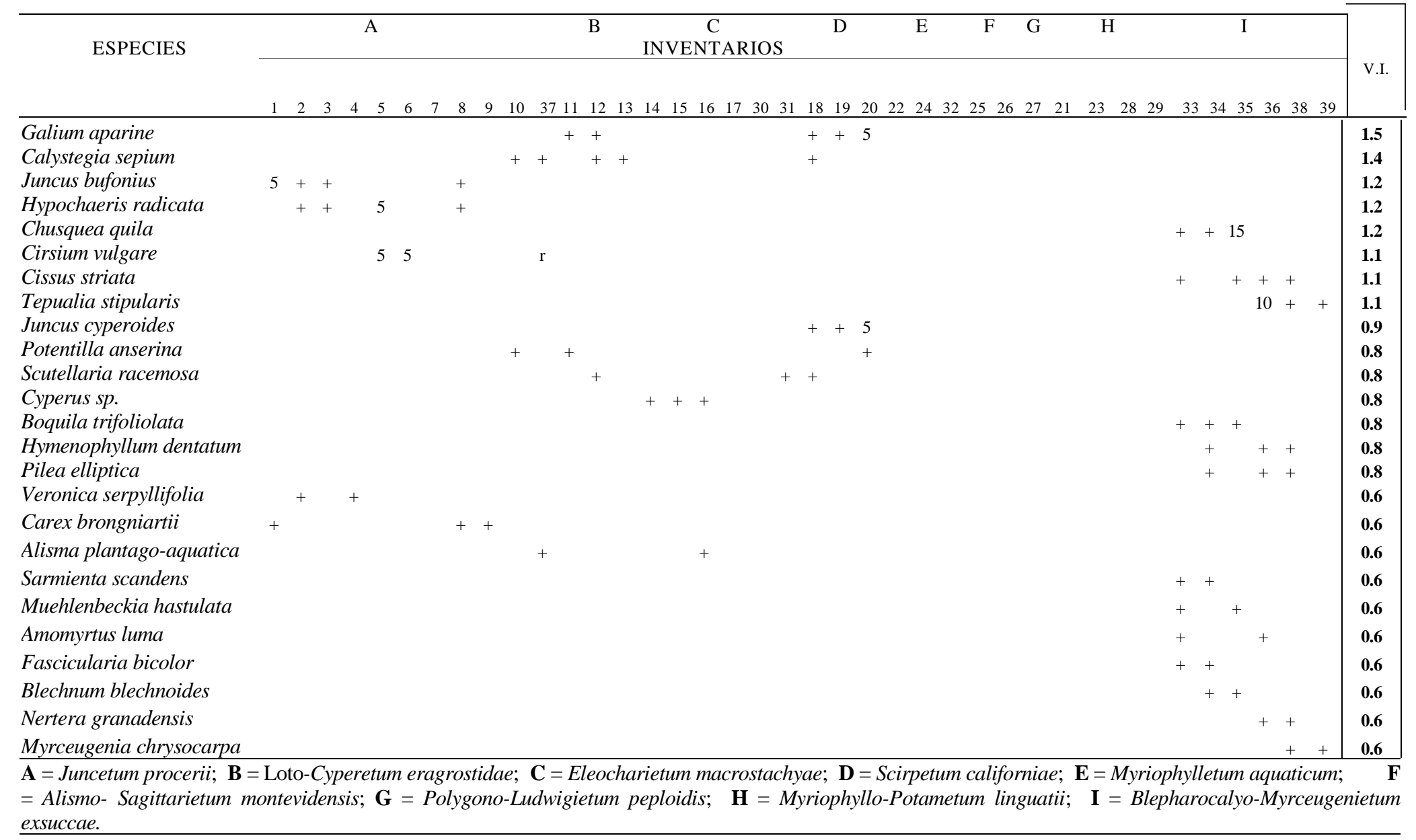


9. Bosque pantanoso de temo y pitra (Blepharocalyo-Myrceugenietum exsuccae Oberdorfer 1960).

Inventarios 33-36 y 38-39. Se le conoce también como pitranto o hualve. Corresponde a una comunidad leñosa perennifolia de tipo azonal que depende más de las condiciones de humedad edáfica que de otro factor ambiental. Se encuentra asociada a los esteros, riachuelos o ríos del sector y a depresiones donde se acumula agua. Las especies dominantes son Myrceugenia exsucca (pitra) y Blapharocalyx crukshanksii (temo), siendo Drimys winteri (canelo) una especie acompañante importante. Presenta gran densidad de individuos y su estrata herbácea es muy pobre debido al exceso de humedad. Se determinaron 32 especies para esta comunidad, donde dominan las nativas, destacando el alto número de epífitas y trepadoras. Es una de las comunidades más fuertemente afectadas por la acción antrópica, ya que ha sido talada intensivamente y los suelos en que prospera están siendo drenados para obtener espacios aptos para la agricultura.

Las nueve asociaciones anteriormente descritas se pueden agrupar en ocho de tipo herbáceas y una leñosa. Las cinco primeras presentan hábitat palustre o de lugares muy húmedos, donde predominan las especies helófitas, las tres siguientes corresponden a hidrófitas típicas y la última corresponde al bosque pantanoso de mirtáceas.

Las siete especies con los mayores valores de importancia en el área, es decir, que presentan alta frecuencia y cobertura son: Myrceugenia exsucca, Eleocharis macrostachya, Scirpus californicus, Juncus procerus, Anagallis alternifolia, Lotus uliginosus y E. pachycarpa.

\section{DISCUSION}

Respecto de la flora, el bajo porcentaje de pteridófitos y la ausencia de gimnospermas en estos humedales (Fig. 1) se podría explicar debido a que su hábitat preferente es el bosque (Godoy et al. 1981). Por su parte, el bajo número de terófitos y caméfitos (Fig. 2) se explica porque estas dos últimas formas de vida representan a climas áridos y a lugares fríos respectivamente (Ramírez 1988), lo que no corresponde a las condiciones climáticas del lugar. Asimismo, el alto porcentaje de especies introducidas en el sector (Fig. 3), junto con el predominio de hemicriptófitos, es una muestra de la presión antrópica a que han estado sometidos estos humedales (Hauenstein et al. 1988).

La riqueza florística de los humedales de Toltén es alta (84 spp.), y supera levemente a la reportada por Ramírez et al. (1991) para el Santuario de la Naturaleza "Río Cruces" en Valdivia (80 spp.), el que a su vez presenta un porcentaje de especies alóctonas superior (32,5\%), lo cual indica una mayor actividad antrópica, entre ellas el gran flujo de turistas.

En relación a la vegetación, habitualmente las comunidades acuáticas presentan una clara zonación o ubicación en franjas bien delimitadas a orillas de cursos de agua (Ramírez et al. 1982; San Martín et al. 1999). La zona más alejada del curso de agua la ocupa el Juncetum procerii, le siguen el Loto-Cyperetum eragrostidae, el AlismoSagittarietum y el Scirpetum californiae. Ya en el agua aparecen las hidrófitas típicas como el Myriophylletum aquaticum que corresponde a una comunidad sumergida, el Myriophyllo-Potametum linguatii y Polygono-Ludwigietum peploidis, ambas de hoja natante. Hay que mencionar además que en el caso de Ludwigia peploides, especie principal de esta última asociación, se puede comportar también como un helófito, presentando incluso heterofilia. Para el caso del Alismo-Sagittarietum, se puede comportar a su vez como una comunidad netamente acuática.

Riffo \& Villarroel (2000) se refieren a esta zonación en franjas de la vegetación acuática como "zonas de vegetación", en las que mencionan, para los humedales del sector los Batros en la comuna de San Pedro de la Paz, provincia de Concepción, las siguientes zonas: a) macrófitas ribereñas, b) pajonales cerrados semi-sumergidos, c) pajonales abiertos, d) pastizales húmedos $\mathrm{y}$, d) bosques hidrófilos. En estas categorías se pueden incluir todas las asociaciones mencionadas en este estudio.

Al comparar estos resultados con la vegetación riparia encontrada por San Martín et al. (2001) en la V Región de Valparaíso, no hay coincidencia con las cuatro asociaciones determinadas por estos autores, en las cuales hay un claro predominio de especies alóctonas sobre las nativas y muchas de ellas adaptadas a soportar altos 
grados de contaminación o eutrofización acuática, situación común en cuerpos de agua cercanos a las ciudades. La situación en los humedales de Toltén es un tanto diferente, ya que aunque existe actividad antrópica, la densidad poblacional del área es baja y no se aprecian signos evidentes de contaminación. Por su parte, San Martín et al. (1993) para el Santuario de la Naturaleza Río Cruces en Valdivia describe 13 asociaciones, en las cuales están incluidas ocho de las mencionadas en este trabajo, con excepción del Eleocharietum macrostachyae. Lo anterior indica que estas comunidades se repiten en hábitat de características semejantes.

La pradera húmeda de junquillo es una asociación secundaria común en el sur de Chile, que reemplaza al bosque pantanoso de temo y pitra y se utiliza intensivamente para uso ganadero (Ramírez et al. 1983). Igual situación ocurre con la pradera de rume, ya que sus dos especies principales son de gran palatabilidad para el ganado, por lo que es ramoneada fuertemente. Presenta un gran potencial como pradera natural al servicio de la actividad agroganadera.

El bosque de temo y pitra es una de las comunidades más fuertemente afectadas por la acción antrópica en el lugar, ya que ha sido talado intensivamente y los suelos en que prospera están siendo drenados para obtener espacios aptos para la agricultura, existiendo incluso programas regionales con subsidio estatal para cumplir dicho objetivo, lo que representa un serio atentado a la rica biodiversidad de estos ecosistemas (Ojeda 1998). Esta asociación boscosa, junto con el totoral, constituyen un hábitat importante para el refugio y nidificación de la fauna existente en el área, entre las que se cuentan especies con problemas de conservación, como el huillín o nutria de río (Lontra provocax), güiña (Felis guigna), torcaza (Columba araucana) y quique (Galictis cuja) (Hauenstein et al. 2001).

Según San Martín et al. (1993) el totoral es la asociación palustre más abundante en el centrosur de Chile, lo que coincide con lo determinado en el área estudiada. El aporte de gran cantidad de necromasa al sustrato y la regeneración permanente de sus culmos es lo que da a la totora un alto grado de competitividad y justifica su dominancia en los cuerpos de agua en que habita (Ramírez \& Añazco 1982).

Por su parte, las comunidades de hidrófitas típicas se caracterizan por su gran capacidad de retención de partículas en suspensión y de absorción y acumulación de sustancias, por lo que cumplen un importante rol en la purificación de las aguas (Ramírez et al. 1982; Rodríguez \& Dellarossa 1998), también pueden cumplir un importante rol como indicadoras de contaminación en los cuerpos de agua en que habitan (Hauenstein et al. 1996, 1999a).

Es importante mencionar que varias de las especies palustres, como Juncus procerus, Scirpus californicus, Cyperus eragrostis y Carex acutata, se utilizan habitualmente en artesanía popular, actividad que no se ha desarrollado en el área estudiada, por lo que ésta podría ser para las comunidades humanas aledañas un importante factor de desarrollo (Hauenstein et al. 2001).

Considerando la riqueza florística de estos humedales, el carácter de refugio y nidificación para la fauna silvestre y su gran potencial como polo de desarrollo para las comunidades humanas ad-yacentes, se justifican plenamente las propuestas de conservación y/o manejo de estos ecosistemas, que han realizado distintas organizaciones tanto públicas como privadas.

\section{AGRADECIMIENTOS}

Este estudio fue posible gracias al apoyo financiero de la Dirección de Investigación de la Universidad Católica de Temuco, a través del proyecto DIUCT $\mathrm{N}^{\circ}$ 99-4-04.

\section{BIBLIOGRAFIA}

AÑAzco, N. 1978. Estudios ecológicos en pobla-ciones de Scirpus californicus (Mey.) Steud. en la provincia de Valdivia, Chile. Tesis de Grado. Escuela de Biología y Química, Universidad Austral de Chile, Valdivia.

Barrera, J. \& C. Ramírez. 1986. Origen, características y aprovechamiento de los bañados del sur de Chile. Versiones Abreviadas II Encuentro Científico sobre el Medio Ambiente Chileno, Talca, 1: 52-56.

Braun-Blanquet, J. 1964. PflanzensoziologieGrundzuge der Vegetationskunde. Springer Verlag, Wien. 865 pp.

Conaf \& Conama. 1999. Catastro y evaluación de recursos vegetacionales nativos de Chile. Informe regional IX Región. Proyecto CONAF 
- CONAMA - BIRF. Santiago, Chile. 90 pp.

Contreras, D., M. Verdugo, J. San Martín \& C. Ramírez. 1991. Flora y vegetación de las praderas húmedas de Chivilcán (Cautín, Chile). Actas II Congreso Internacional de Gestión en Recursos Naturales 2: 438-455.

Di CAstri, F. \& E. Hajek. 1976. Bioclimatología de Chile. P. Universidad Católica de Chile, Santiago. $128 \mathrm{pp}$.

Ellenberg, H. \& D. Mueller-Dombois. 1966. A key to Raunkiaer plant life forms with revised subdivisions. Veröff. Geobot. Inst. ETH Stiftung Rübel, Zurich 37: 56-73.

Godoy, R., C. Ramirez, H. Figueroa \& E. Hauenstein. 1981. Estudios ecosociológicos en pteridófitos de comunidades boscosas valdivianas, Chile. Bosque 4: 12-24.

GonZÁlEZ, G. 1993. Los humedales. Ambientes amenazados y olvidados. Chile Forestal 208:34-35.

Granizo, T. (ED.). 1997. Uso sostenible de humedales en suramérica: una aproximación. UICN-Sur. Quito, Ecuador. 126 pp.

Hauenstein, E., M. González, L. Leiva \& L. Falcón. 1999a. Flora de macrófitos y bioindicadores del lago Budi (IX Región, Chile). Gayana Botanica 56: 53-62.

Hauenstein, E., A. Muñoz-Pedreros, F. Peña \& M. GonZÁLEZ. 2001. Bases para la conservación de los humedales de la costa de Toltén (IX Región). Informe Final Proyecto DIUCT N ${ }^{\circ}$ 99-404. Dirección de Investigación, Universidad Católica de Temuco. $56 \mathrm{pp}+$ anexos.

Hauenstein, E., C. Ramírez, M. Latsague \& D. Contreras. 1988. Origen fitogeográfico y espectro biológico como medida del grado de intervención antrópica en comunidades vegetales. Medio Ambiente 9: 140-142.

Hauenstein, E., A. Muñoz-Pedreros, F. Peña, F. Encina \& M. González. 1999b. Humedales: ecosistemas de alta biodiversidad con problemas de conservación. El Arbol...Nuestro Amigo 13: 8-12.

Hauenstein, E., C. Ramírez, M. González, L. Leiva \& C. SAN Martín. 1996. Flora hidrófila del lago Villarrica (IX Región, Chile) y su importancia como elemento indicador de contaminación. Medio Ambiente 13: 88-96.

Instituto Geográfico Militar. 1986. Geografía de la IX Región. Ediciones del Instituto Geográfico Militar. Santiago, Chile. 250 pp.

Koeppen, W. 1931. Die Klimate der Erde. Grundiss der Klimakunde. 2 Auf. Berlin. 182 pp.

Kusler J.A., W.J. Mitsch \& J.S. Larson. 1994. Humedales. Investigación y Ciencia 210: 6-13.

Marticorena, C. \& M. Quezada. 1985. Catálogo de la flora vascular de Chile. Gayana Botánica 42: 1155.

Matthei, O. 1995. Manual de las malezas que crecen en Chile. Alfabeta Impresores. Santiago, Chile. $545 \mathrm{pp}$.
Medina, R. 1988. Flora y fitosociología del Santuario de la Naturaleza Río Cruces (Valdivia, Chile). Tesis de Grado. Fac. Ciencias Forestales, Universidad Austral de Chile, Valdivia.

Muñoz-Pedreros, A. \& P. Moller (Eds.). 1997. Conservación de humedales. Bases para la conservación de humedales de Chile. Ediciones del Centro de Estudios Agrarios \& Ambientales (CEA). Valdivia, Chile. 95 pp.

MuÑoz, M., H. NúÑEZ \& J. YÁÑEZ (EDs.). 1996. Libro rojo de los sitios prioritarios para la conservación de la diversidad biológica en Chile. Ministerio de Agricultura, Corporación Nacional Forestal. Santiago. 203 pp.

Oberdorfer, E. 1960. Pflanzensoziologishe Studien in Chile: Ein Vergleich mit Europa. Flora et Vegetatio Mundi 2: 1-208.

Ramírez, C. 1988. Formas de vida, fitoclimas y formaciones vegetales. El Arbol...Nuestro Amigo 4: 33-37.

Ramírez, C. \& N. AÑazco. 1982. Variaciones estacionales en el desarrollo de Scirpus californicus, Typha angustifolia y Phragmites communis en pantanos valdivianos, Chile. Agro Sur 10: 11-23.

Ramírez, C. \& E. Stegmeier. 1982. Formas de vida en hidrófitos chilenos. Medio Ambiente 6: 43-54.

Ramírez, C. \& R. Westermeier. 1976. Estudio de la vegetación espontánea del Jardín Botánico de la Universidad Austral de Chile (Valdivia) como ejemplo de tabulación fitosociológica. Agro Sur 4: 93-105.

Ramírez, C., F. Ferriere \& H. Figueroa. 1983. Estudio fitosociológico de los bosques pantanosos templados del sur de Chile. Revista Chilena de Historia Natural 56: 11-26.

Ramírez, C., R. Godoy, D. Contreras \& E. Stegmaier. 1982. Guía de plantas acuáticas y palustres valdivianas. Instituto de Botánica, Facultad de Ciencias, Universidad Austral de Chile. 42 pp.

Ramírez, C., C. San Martín, R. Medina \& D. Contreras. 1991. Estudio de la flora hidrófila del Santuario de la Naturaleza "Río Cruces" (Valdivia, Chile). Gayana Botánica 48: 67-80.

Riffo, R. \& C. Villarroel. 2000. Caracterización de la flora y fauna del humedal Los Batros, comuna de San Pedro de La Paz. Gayana 64 Suplemento: $23-37$.

Rodríguez, R. \& V. Dellarossa. 1998. Plantas vasculares acuáticas en la región del Bío-Bío. Ediciones Universidad de Concepción, Concepción. 38 pp.

San Martín, C., C. Ramírez \& P. OJeda. 1999. Distribución de macrófitos y patrones de zonación ribereña en la cuenca del río Valdivia, Chile. Revista Geográfica de Valparaíso 29-30: 117-126.

San Martín, C., R. Medina, P. OJeda \& C. Ramírez. 1993. La biodiversidad vegetacional del Santuario de la Naturaleza "Río Cruces" (Valdivia, Chile). Acta Botánica Malacitana 18: 259-279. 
Gayana Bot. 59(2), 2002

San Martin, C., C. Ramírez, J. San Martín \& R. VILLASEÑOR. 2001. Flora y vegetación del estero Reñaca (V Región, Chile). Gayana Botánica 58: 31-46.
Wikum, D. \& G.F. Schanholtzer. 1978. Application of the Braun-Blanquet cover-abundance scale for vegetation analysis in land development studies. Environmental Management 2: 323-329.

Fecha de recepción: 20.12.01

Fecha de aceptación: 02.01.03

Fecha de publicación: Abril de 2003. 